\title{
Human amnion-derived mesenchymal stem cells induced osteogenesis and angiogenesis in human adipose-derived stem cells via ERK1/2 MAPK signaling pathway
}

\author{
Yuli Wang ${ }^{1,3}$, Xichen Chen ${ }^{4}$, Ying Yin ${ }^{5} \mathcal{E}$ Song $\mathrm{Li}^{1,2, *}$ \\ ${ }^{1}$ Jiangsu Key Laboratory of Oral Diseases, Nanjing Medical University, Nanjing 210029, Departments of ${ }^{2}$ Periodontology, ${ }^{3}$ Oral and \\ Maxillofacial Surgery, Affiliated Hospital of Stomatology, Nanjing Medical University, Nanjing 210029, ${ }^{4}$ Analysis Center, Nanjing Medical \\ University, Nanjing 211166, ${ }^{5}$ Nanjing Stomatological Hospital, Medical School of Nanjing University, Nanjing 210008, China
}

\begin{abstract}
Mesenchymal stem cells (MSCs) have shown great potential in treating bone deficiency. Human adipose-derived stem cells (HASCs) are multipotent progenitor cells with multi-lineage differentiation potential. Human amnion-derived mesenchymal stem cells (HAMSCs) are capable of promoting osteogenic differentiation of MSCs. In this study, we investigated the effect of HAMSCs on HASCs by a transwell co-culture system. HAMSCs promoted proliferation, osteogenic differentiation, angiogenic potential and adiponectin (APN) secretion of HASCs. Moreover, the positive effect of HAMSCs was significantly inhibited by U0126, a highly selective inhibitor of extracellular signaling-regulated kinase 1/2 (ERK1/2) mitogen-activated protein kinase (MAPK) signaling pathway. These observations suggested that HAMSCs induced bone regeneration in HASCs via ERK1/2 MAPK signaling pathway. [BMB Reports 2018; 51(4): 194-199]
\end{abstract}

\section{INTRODUCTION}

Bone deficiency is an emerging risk for patients requiring dental implants since it results in the failure of restoring oral function and is spontaneous if left untreated (1). Recently, seed cells have shown enormous potential in the field of tissue engineering (2). Adipose is considered as an ideal source of cells for tissue regeneration (3). Human adipose-derived stem cells (HASCs) are associated with minimal donor site morbidity, low immunogenicity, capacity for self-renewal and

${ }^{*}$ Corresponding author. Tel: +8602585031826; Fax: +860258503 1826; E-mail: lisongnjmu@163.com

https://doi.org/10.5483/BMBRep.2018.51.4.005

Received 11 January 2018, Revised 2 February 2018, Accepted 6 February 2018

Keywords: Angiogenesis, ERK1/2 MAPK signaling pathway, Human adipose-derived stem cells, Human amnion-derived mesenchymal stem cells, Osteogenesis multi-lineage differentiation (4). For instance, growth factors and cytokines such as bone morphogenetic protein 2 (BMP-2) and adiponectin (APN), have been used to enhance the osteogenic differentiation of HASCs (5). However, individual differences, complicated synthesis, easy degradation, and limited availability restrict their application.

Human amniotic membrane (AM) is an abundant tissue with substantial benefits (6). Human amnion-derived mesenchymal stem cells (HAMSCs), derived from AM, have low antiinflammatory properties and fewer ethical concerns as compared to other sources of mesenchymal stem cells (MSCs) $(7,8)$. Our previous studies demonstrated that HAMSCs promoted osteogenic differentiation of human bone marrow mesenchymal stem cells (HBMSCs) and provided a conducive environment against the oxidative stress and lipopolysaccharide (LPS)-induced bone deficiency by influencing mitogenactivated protein kinase (MAPK) signaling pathway (9-12). We, therefore, hypothesized the role of HAMSCs and MAPK signaling pathway in regulating the differentiation of HASCs.

APN, one of the most abundant adipocytokines secreted by adipocytes, facilitated the osteoblastic differentiation of mesenchymal progenitor cells (13) and bone formation in animal models (14). Furthermore, MAPK signaling pathway is essential for the regulation of fatty acid metabolism by APN (15). However, whether the interaction between APN and MAPK signaling pathway is involved in the differentiation of HASCs regulated by HAMSCs remains unclear.

In this study, a transwell co-culture system was used to determine the in vitro effect of HAMSCs on osteogenesis and angiogenesis in HASCs. HAMSCs not only promoted the proliferation, osteoblastic differentiation, and angiogenic potential of HASCs but also increased APN level in a dose-dependent manner. Extracellular signaling-regulated kinase 1/2 (ERK1/2) MAPK signaling pathway played a key role in these processes. 


\section{RESULTS}

\section{HAMSCs promoted HASC proliferation}

Cell cycle fractions (G0, G1, S, and G2M phases) were measured by flow cytometry on day 3 . The S-phase checkpoints in HASCs were remarkably accelerated with the HASCs:HAMSCs ratio in co-culture groups (Fig. 1A). These results showed that HAMSCs were capable of promoting HASC proliferation in a dose-dependent manner at the early stage of differentiation.

\section{HAMSCs promoted osteoblastic differentiation in HASCs} The culture surface stained positively for extracellular matrix (ECM) was measured after 21 days. In contrast to the HAMSC groups, higher level of mineralization was observed in HASCs, and the proportion of ECM increased with the HASCs:HAMSCs ratio (Fig. 1B). Osteoblast-related mRNA expression and alkaline phosphatase (ALP) activity in HASCs also gradually increased with the HASCs:HAMSCs ratio as compared to the HAMSC groups (Fig. 1C and D). These findings indicated that HAMSCs promoted osteoblastic differentiation of HASCs in the co-culture system.

\section{HAMSCs promoted APN secretion and angiogenic potential of HASCs}

The APN concentration was analyzed after 14 days. As compared to the single-culture groups, the APN concentration in HASCs/HAMSCs groups were remarkably accelerated with the HASCs:HAMSCs ratio (Fig. 1E). The angiogenic potential of HASCs was further measured by vascular endothelial growth factor (VEGF) enzyme-linked immunosorbent assay (ELISA) on day 14 and tube formation assay of human umbilical vein endothelial cells (HUVECs) at $24 \mathrm{~h}$. The HASCs/HAMSCs groups secreted significantly higher levels of VEGF than the single-culture groups, and the VEGF level gradually increased with HASCs:HAMSCs ratio (Fig. 1F). The HUVEC tube formation assay suggested that the addition of culture supernatant from HASCs/HAMSCs groups contributed to the formation of new tube structures. Extensive tube structures were observed, which increased with HASCs:HAMSCs ratio (Fig. 1G). These results suggested that HAMSCs enhanced APN secretion and angiogenic potential of HASCs.

\section{ERK1/2 MAPK signaling pathway was essential for HAMSC-driven osteogenesis}

We systematically investigated the role of MAPK signaling pathway in HAMSC-driven differentiation. In contrast to the single-culture groups, the ERK $1 / 2$ phosphorylation level in the HASCs/HAMSCs groups gradually increased with HASCs:HAMSCs ratio, while neither p38 nor JNK phosphorylation appeared to be involved (Fig. 2A). The transcriptional expression of runt-related transcription factor 2 (RUNX2), which is essential for osteoblastic differentiation and subsequent bone formation, was also up-regulated by increased HASCs:HAMSCs ratio. Further, U0126 was used to explore whether the effect of HAMSCs was ERK1/2-dependent. U0126 remarkably inhibited ERK1/2 phosphorylation and RUNX2 expression in HASCs with HAMSC treatment (Fig. 2B). Moreover, the effect of HAMSCs in promoting osteoblastic differentiation in HASCs was counteracted by U0126, based on an appropriate HASCs:HAMSCs ratio (Fig. 3A, B and C). These results highlighted the role of ERK1/2 MAPK signaling pathway in HAMSC-driven osteogenesis.

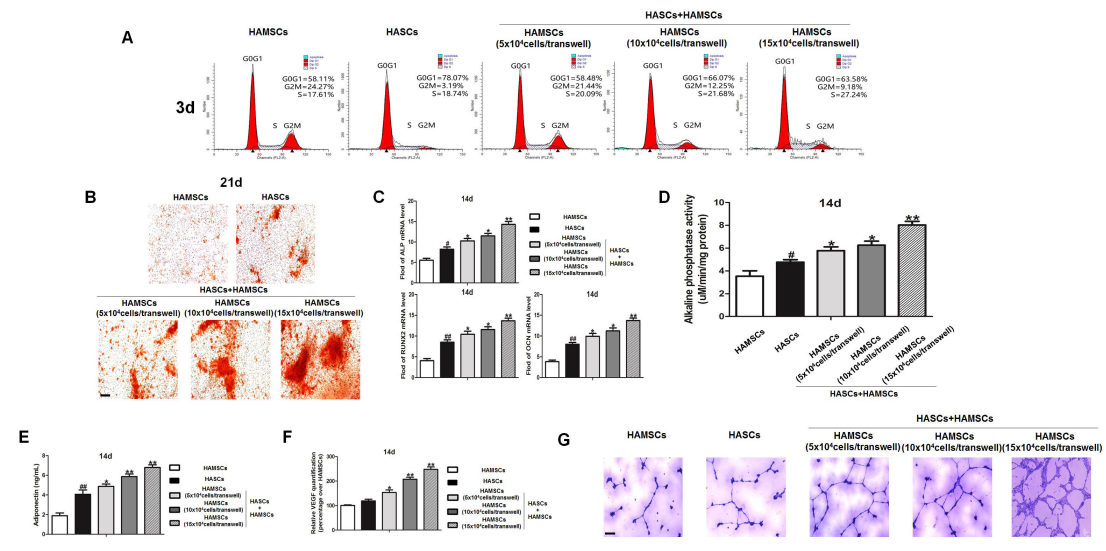

Fig. 1. Effect of HAMSCs on HASCs. (A) Cell cycle fractions (G0, G1, S, and G2M phases) were measured by flow cytometry on day 3 . (B) Mineralized matrix deposition was measured on day 21 by Alizarin red S. (C) The mRNA expression of ALP, RUNX2, and OCN was analyzed on day 14 by RT-PCR. GAPDH was used as the internal control. (D) ALP activity was measured on day 14 using ALP assay kit. (E) APN concentration was measured on day 14 using a human APN ELISA kit. (F) The VEGF level was measured by human VEGF ELISA kit on day 14. (G) The tube formation of HUVECs was analyzed at $24 \mathrm{~h}$. Bar: $300 \mu \mathrm{m}$. ${ }^{\#} \mathrm{P}<0.05$ and ${ }^{\# \#} \mathrm{P}<0.01$ versus the HAMSCs group; $* P<0.05$ and $* * P<0.01$ versus the HASCs group. 


\section{ERK1/2 MAPK signaling pathway played a key role in HAMSC-driven APN secretion and angiogenesis}

To further confirm the role of ERK1/2 MAPK signaling pathway in APN secretion and angiogenesis driven by HAMSCs, we detected the APN concentration, VEGF level and vascular network structures with or without U0126. U0126 significantly down-regulated APN concentration and VEGF level based on an appropriate HASCs:HAMSCs ratio (Fig. 3D and E). Meanwhile, fewer vascular networks were formed by HUVECs after treatment with U0126 (Fig. 3F). These results suggested that HAMSCs promoted APN secretion and angiogenesis through ERK1/2 MAPK signaling pathway.

\section{DISCUSSION}

Tissue engineering using suitable seed cells promises to be a potential method for resolving bone deficiency in stomatological diseases (16). HASCs, obtained from adipose tissue, exhibit

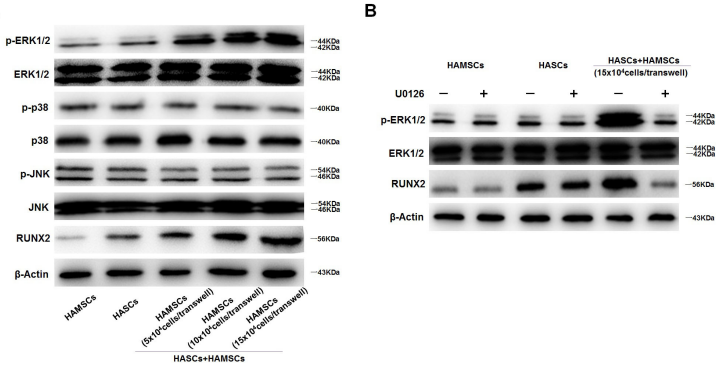

Fig. 2. Expression of relative proteins in HAMSCs and HASCs cultured with or without U0126 on day 14. (A) Protein expression levels of p-ERK1/2, ERK1/2, p-p38, p38, p-JNK, JNK and RUNX2 were determined by Western blot, $\beta$-actin served as an internal control. (B) Protein expression levels of $p$-ERK1/2, ERK1/2 and RUNX2 were determined by Western blot, $\beta$-actin served as an internal control. enormous potential for clinical translation into regeneration therapies, and as seed cells for repairing damaged body parts (17). However, their mild osteogenic differentiation capability limits their application in functional bone reformation, and becomes a major challenge in tissue engineering $(18,19)$.

Previous studies have demonstrated that HAMSC was a potential strategy for bone-destructive processes due to its effect on other cells. In this study, we constructed a transwell co-culture system to evaluate the in vitro effect of HAMSCs on HASCs. HAMSCs enhanced the proliferation of HASCs on day 3, which was confirmed by flow cytometry. Furthermore, HAMSCs could promote mineralized matrix formation, early osteogenic markers expression and ALP activity in HASCs in a dose-dependent manner. These results indicated that HAMSCs can improve HASC osteogenesis.

Bone is a vital organ that needs microvascular networks to supply oxygen and nutrients to maintain normal metabolism (20). Since osteogenic factors secreted by revascularization are necessary for initiating bone formation, angiogenesis plays a key role in tissue regeneration $(21,22)$. Previous studies suggested that both HAMSCs and HASCs showed desirable angiogenic properties $(23,24)$. Therefore, we hypothesized the enhanced angiogenic potential of HASCs treated with HAMSCs. In this study, abundant tube structures were formed by HUVECs after treatment with culture supernatants from HASCs/HAMSCs groups. Higher level of VEGF, which could be released by MSCs and was indispensable in angiogenesis (25), was found in the HASCs/HAMSCs groups. These findings suggested that HAMSCs enhanced the angiogenic potential of HASCs.

After establishing that HAMSCs provided a conducive environment to regulate the proliferation, and osteogenic and angiogenic differentiation of HASCs, we further investigated the cytokines involved in this process. APN, a highly abundant adipocytokine specifically expressed in differentiated adipocytes $(26,27)$, was up-regulated by HAMSCs in a dose-dependent manner. APN plays a potential role in bone metabolism by activating osteoblasts and inhibiting M-CSF and RANKL-

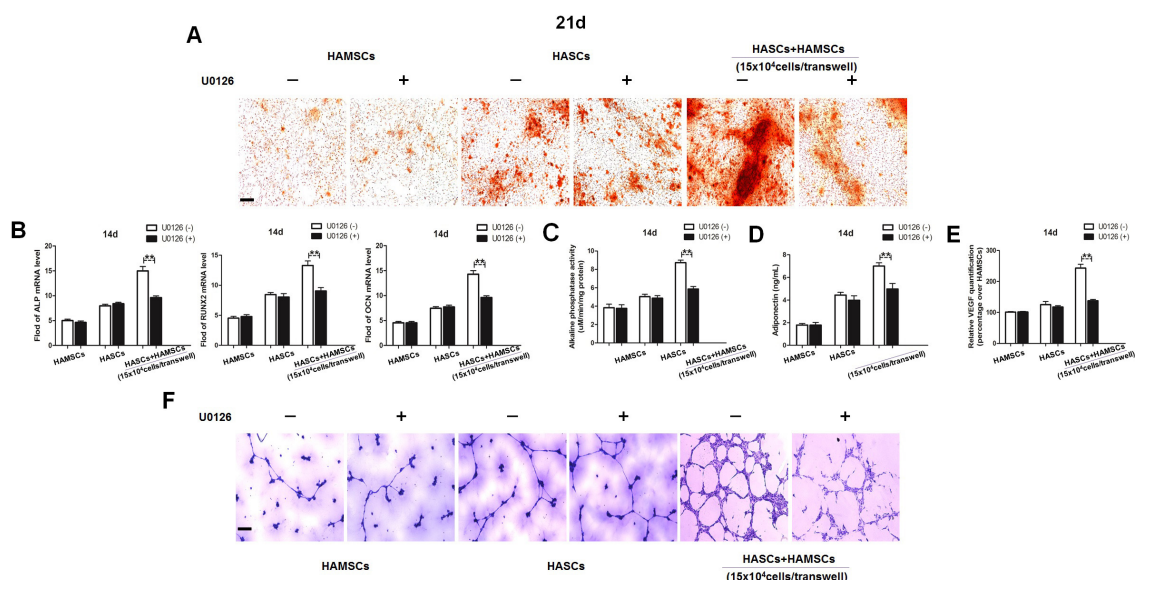

Fig. 3. Effect of U0126 on osteoblastic differentiation, APN secretion and angiogenic potential. (A) Mineralized matrix deposition was measured on day 21 by Alizarin red S. (B) The mRNA expression of ALP, RUNX2, and OCN was analyzed on day 14 by RT-PCR. GAPDH was used as the internal control. (C) ALP activity was measured on day 14 using ALP assay kit. (D) APN concentration was measured on day 14 by a human APN ELISA kit. (E) The VEGF level was measured by human VEGF ELISA kit on day 14. (F) The tube formation of HUVECs was analyzed at $24 \mathrm{~h}$. Bar: $300 \mu \mathrm{m}$. **P $<0.01$. 
induced osteoclast differentiation (28). Studies on the effect of APN on HASCs have shown direct evidence that APN enhanced osteogenic differentiation of HASCs (29). Our results indicated that HAMSC-mediated osteogenesis might be associated with increased APN secretion.

The main aim of this study was to investigate the underlying mechanism engaged by HAMSCs. The osteogenic and angiogenic differentiation of MSCs is regulated by multiple signaling pathways, including Wnt- $\beta$-Catenin, Hedgehog, Insulin Receptor and MAPK (30-32). In this study, the MAPK signaling pathway was investigated. We found that ERK $1 / 2$ signaling pathway, the phosphorylation of which is a crucial trigger of osteogenic differentiation (33), was activated by HAMSCs in a dose-dependent manner, while neither the p38 nor JNK pathways appeared to be involved. The transcriptional activity of RUNX2, which is essential for subsequent bone formation and expression of osteogenic genes (34), was also increased by HAMSCs. Furthermore, U0126 significantly inhibited the positive effect of HAMSCs in promoting osteogenic and angiogenic differentiation of HASCs by suppressing the ERK1/2 MAPK signaling pathway. Interestingly, APN concentration was down-regulated by U0126, which suggested that HAMSC-driven APN secretion was also ERK1/2-dependent. Therefore, we will examine the relationship between HAMSCs, ERK1/2 MAPK signaling pathway and APN in the future.

In summary, our data demonstrated that HAMSCs could promote osteogenic and angiogenic differentiation of HASCs in vitro, which was mediated by phosphorylation of ERK $1 / 2$ MAPK signaling pathway. These findings highlighted the molecular mechanism mediated by HAMSCs, and may facilitate the application of HASCs in tissue engineering.

\section{MATERIALS AND METHODS}

\section{Chemicals and reagents}

Fetal bovine serum (FBS), trypsin-ethylenediaminetetraacetic acid (EDTA), phosphate-buffered saline (PBS), $\alpha$-minimum essential medium ( $\alpha M E M)$ and penicillin G-streptomycin sulfate were purchased from Gibco ${ }^{\circledR}$ Life Technologies. The Alizarin red $\mathrm{S}(\mathrm{pH} 4.4), \mathrm{ALP}$ and bicinchoninic acid (BCA) assay kits were purchased from Jiancheng Corp (Nanjing, China). Dexamethasone, $\beta$-glycerophosphate ascorbic acid and dimethyl sulfoxide (DMSO) were purchased from Sigma-Aldrich (St. Louis, MO). Six-well culture plates and transwells (6-well Millicell Hanging Cell Culture Inserts, 0.4 $\mu \mathrm{m}, \mathrm{PET}$ ) were purchased from Millipore ${ }^{\mathbb{R}}$ (Bedford, MA, USA). Growth factor-reduced Matrigel was purchased from BD Bioscience (San Diego, CA). EGM-2 Bullet kit was purchased from Lonza (Walkersville, MD, USA). APN (human) ELISA kit (K4901-100) was purchased from Biovision ${ }^{\circledR}$ (Germany). VEGF (human) ELISA kit was purchased from R\&D Systems (Minneapolis, MN, USA). The antibodies specific for human CD29, CD31, CD34, CD44, CD45, CD73, CD90, CD105, CD166, and HLA-DR were purchased from R\&D Systems
(Boston, MA, USA). The mouse anti-rabbit IgG (L27A9) mAb, phospho-p38 (p-p38) MAPK (Thr180/Tyr182) (D3F9) rabbit mAb, p38 MAPK (D13E1) rabbit mAb, phospho-SAPK/JNK (p-JNK) (Thr183/Tyr185) (81E11) rabbit mAb, SAPK/JNK (JNK) antibody (\#9252), phospho-p44/42 (p-ERK1/2) MAPK rabbit mAb, p44/42 MAPK (ERK1/2) rabbit mAb, RUNX2 (D1L7F) rabbit mAb, $\beta$-actin (13E5) rabbit mAb, and U0126 (\#9903) were purchased from Cell Signaling Technology (Danvers, MA, USA). Triton X-100, RIPA buffer, and Trizol were purchased from Beyotime (Shanghai, China). Other reagents used were of the highest commercial grade available.

\section{Cells and cell culture}

HASCs were obtained from ScienCell Co. (San Diego, CA, USA). Passage three HASCs were characterized by flow cytometry (BD Biosciences, Franklin Lakes, NJ, USA) after staining with monoclonal antibodies specific for human CD29, CD31, CD34, CD45, CD44, CD73, CD105, CD166, and HLA-DR (Fig. 4A). HAMSCs were prepared as previously described (35), and third-passage HAMSCs were characterized using specific antibodies (Fig. 4B). Cell types were cultured in aMEM supplemented with $100 \mathrm{U} / \mathrm{L}$ penicillin, $100 \mathrm{mg} / \mathrm{L}$ streptomycin, and $10 \%$ FBS in a humidified atmosphere of $5 \%$ $\mathrm{CO}_{2}$ at $37^{\circ} \mathrm{C}$. Cells from passages 3-6 were used, and culture medium was changed every three days. The study protocols were approved by the Ethics Committee of the School of Stomatology, Nanjing Medical University, China (NO.PJ2013037-001). Informed consent was obtained from all the study participants.

\section{Transwell co-culture system}

A transwell co-culture system was established to measure the effect of HAMSCs on HASCs, as previously described (10).

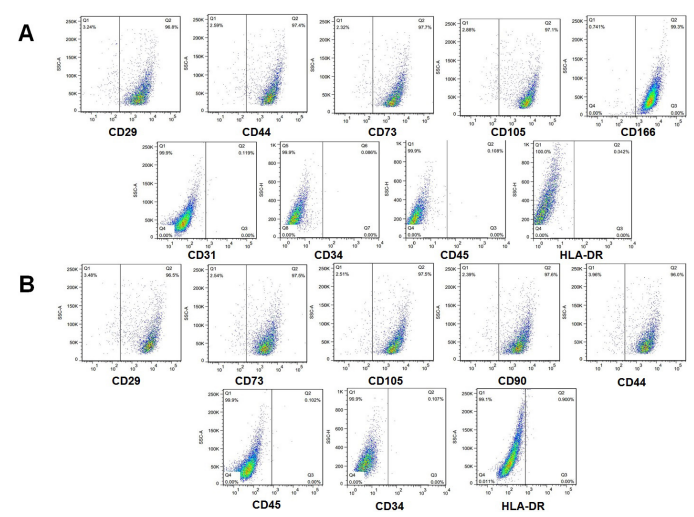

Fig. 4. Characterization of HASCs and HAMSCs. (A) Flow cytometric analysis confirmed that HASCs were positive for CD29, CD44, CD73, CD105 and CD166, but negative for CD31, CD34, CD45 and HLA-DR. (B) Flow cytometric analysis confirmed that HAMSCs were positive for CD29, CD73, CD105, CD90 and CD44, but negative for CD45, CD34 and HLA-DR. 
HASCs were seeded at an initial cell density of $5 \times 10^{4} \mathrm{cells} / \mathrm{cm}^{2}$ and HAMSCs were seeded at increasing HASCs:HAMSCs ratios $\left(5 \times 10^{4}\right.$ cells/transwell, $10 \times 10^{4}$ cells/transwell and $15 \times 10^{4}$ cells/transwell). HASCs with HAMSCs served as the treatment groups, while HAMSCs and HASCs alone served as the control groups.

\section{Cell proliferation assay}

The proliferation of HASCs co-cultured with HAMSCs was determined by flow cytometry, as previously described (11). Cell cycle fractions (G0, G1, S, and G2M phases) were processed on day 3 using CellQuest Pro software (BD Biosciences).

\section{Osteoblastic differentiation}

Osteoblastic differentiation of both cell types was induced using osteogenic medium (OS) containing $10 \mathrm{mM}$ $\beta$-glycerophosphate, $100 \mathrm{nM}$ ascorbic acid, and $100 \mathrm{nM}$ dexamethasone. The effect of HAMSCs on HASC osteoblastic differentiation was analyzed by ALP activity and mineralized matrix formation, which was followed by Western Blotting and quantitative real-time reverse transcription polymerase chain reaction (RT-PCR). U0126, a highly selective inhibitor of ERK1/2 MAPK signaling, was prepared in DMSO and used at a concentration of $20 \mu \mathrm{M}$, as previously described (36).

\section{ALP activity assay and mineralized matrix formation}

ALP activity and mineralized matrix formation were measured as previously described (12). ALP activity was expressed as micromoles of reaction product per minute per total protein. Bone nodules were visualized using an inverted microscope (Carl Zeiss Ag, Oberkochen, Germany) and five images were captured for each group.

\section{RT-PCR and western blotting}

RT-PCR and Western Blotting were performed as previously described (12). The primer sequences used for RT-PCR were as follows: human RUNX2 (forward, 5'-CCGCACAACCGCACCAT3'; reverse, 5'-CGCTCCGGCCCACAAATCTC-3'), human osteocalcin (OCN) (forward, 5'-CATGAGAGCCCTCACA-3'; reverse, 5'-AGAGCGACACCCTAGAC-3'), human ALP (forward, 5'-TGGAGGTTCAGAAGCTCAACACCA-3'; reverse, 5'-ATCTC GTTCTCTGAGTACCAGTC-3'), and human glyceraldehyde-3phosphate dehydrogenase (GAPDH) (forward, 5'-GGGCTGC TITTAACTCTGGT-3'; reverse, 5'-GCAGGTITTCTAGACGG-3'). GAPDH expression was analyzed to normalize target gene expression. The primary antibodies used for Western Blotting were as follows: RUNX2 (1:1000), ERK1/2 (1:500), p-ERK1/2 (1:500), p38 (1:1000), p-p38 (1:1000), JNK (1:500), and p-JNK (1:500). $\beta$-actin (1:500) served as an internal control.

\section{APN and VEGF quantification, and HUVEC tube formation assay}

The culture supernatants of the control and treatment groups were collected from the co-culture system after 14 days, and assayed to measure the levels of APN and VEGF. Human APN and VEGF ELISA kits were used according to the manufacturer's instructions. The VEGF values were expressed as fold changes over that of the control (HASCs treated without HAMSCs).

Third-passage HUVECs were purchased from the China Infrastructure of Cell Line Resources (Beijing, China), and the tube formation assay was performed as previously described (37). Tube formation was visualized using an inverted microscope (Carl Zeiss AG, Oberkochen, Germany) and five images were captured for each group.

\section{Statistical analysis}

All experiments were independently repeated at least three times. Statistically significant differences $(P<0.05)$ were evaluated using Student's t-test or ANOVA. All statistical analyses were performed using SPSS 19.0 software (SPSS Inc., Chicago, IL, USA).

\section{ACKNOWLEDGEMENTS}

This study was supported by the Priority Academic Program Development of Jiangsu Higher Education Institutions (PAPD; 2014-37).

\section{CONFLICTS OF INTEREST}

The authors have no conflicting interests.

\section{REFERENCES}

1. Kassebaum NJ, Bernabe E, Dahiya M, Bhandari B, Murray CJ and Marcenes W (2014) Global burden of severe periodontitis in 1990-2010: a systematic review and meta-regression. J Dent Res 93, 1045-1053

2. Liu Y, Sun H, Hu M et al (2017) Human Corneal Endothelial Cells Expanded In Vitro Are a Powerful Resource for Tissue Engineering. Int J Med Sci 14, 128-135

3. Zuk PA, Zhu M, Ashjian P et al (2002) Human adipose tissue is a source of multipotent stem cells. Mol Biol Cell 13, 4279-4295

4. Hong JM, Kim BJ, Shim JH et al (2012) Enhancement of bone regeneration through facile surface functionalization of solid freeform fabrication-based three-dimensional scaffolds using mussel adhesive proteins. Acta Biomater 8, 2578-2586

5. Park KH, Kim H, Moon S and Na K (2009) Bone morphogenic protein-2 (BMP-2) loaded nanoparticles mixed with human mesenchymal stem cell in fibrin hydrogel for bone tissue engineering. J Biosci Bioeng 108, 530-537

6. Sheridan RL and Moreno C (2001) Skin substitutes in burns. Burns 27, 92

7. Alviano F, Fossati V, Marchionni C et al (2007) Term Amniotic membrane is a high throughput source for 
multipotent Mesenchymal Stem Cells with the ability to differentiate into endothelial cells in vitro. BMC Dev Biol 7,11

8. Wolbank S, Peterbauer A, Fahrner M et al (2007) Dose-dependent immunomodulatory effect of human stem cells from amniotic membrane: a comparison with human mesenchymal stem cells from adipose tissue. Tissue Eng 13, 1173-1183

9. Wang $Y$, Yin $Y$, Jiang $F$ and Chen $N$ (2015) Human amnion mesenchymal stem cells promote proliferation and osteogenic differentiation in human bone marrow mesenchymal stem cells. J Mol Histol 46, 13-20

10. Wang $Y$, Jiang F, Liang $Y$, Shen $M$ and Chen N (2016) Human Amnion-Derived Mesenchymal Stem Cells Promote Osteogenic Differentiation in Human Bone Marrow Mesenchymal Stem Cells by Influencing the ERK1/2 Signaling Pathway. Stem Cells Int 2016, 4851081

11. Wang Y, Ma J, Du Y, Miao J and Chen N (2016) Human Amnion-Derived Mesenchymal Stem Cells Protect Human Bone Marrow Mesenchymal Stem Cells against Oxidative Stress-Mediated Dysfunction via ERK1/2 MAPK Signaling. Mol Cells 39, 186-194

12. Wang $\mathrm{Y}, \mathrm{Wu} \mathrm{H}$, Shen $\mathrm{M}$, Ding $\mathrm{S}$, Miao J and Chen $\mathrm{N}$ (2017) Role of human amnion-derived mesenchymal stem cells in promoting osteogenic differentiation by influencing p38 MAPK signaling in lipopolysaccharide-induced human bone marrow mesenchymal stem cells. Exp Cell Res 350, 41-49

13. Lee HW, Kim SY, Kim AY, Lee EJ, Choi JY and Kim JB (2009) Adiponectin stimulates osteoblast differentiation through induction of COX2 in mesenchymal progenitor cells. Stem Cells 27, 2254-2262

14. Jiang X, Song D, Ye B et al (2011) Effect of intermittent administration of adiponectin on bone regeneration following mandibular osteodistraction in rabbits. J Orthop Res 29, 1081-1085

15. Park M, Wu D, Park T et al (2013) APPL1 transgenic mice are protected from high-fat diet-induced cardiac dysfunction. Am J Physiol Endocrinol Metab 305, E795-804

16. Corbella S, Taschieri S, Francetti L, Weinstein R and Del Fabbro M (2017) Histomorphometric Results After Postextraction Socket Healing with Different Biomaterials: A Systematic Review of the Literature and Meta-Analysis. Int J Oral Maxillofac Implants 32, 1001-1017

17. Gimble JM, Katz AJ and Bunnell BA (2007) Adiposederived stem cells for regenerative medicine. Circ Res $100,1249-1260$

18. Tsuji W, Rubin JP and Marra KG (2014) Adipose-derived stem cells: Implications in tissue regeneration. World J Stem Cells 6, 312-321

19. Ruetze M and Richter W (2014) Adipose-derived stromal cells for osteoarticular repair: trophic function versus stem cell activity. Expert Rev Mol Med 16, e9

20. Kempen DH, Lu L, Heijink A et al (2009) Effect of local sequential VEGF and BMP-2 delivery on ectopic and orthotopic bone regeneration. Biomaterials 30, 2816-2825

21. Kanczler JM and Oreffo RO (2008) Osteogenesis and angiogenesis: the potential for engineering bone. Eur Cell Mater 15, 100-114

22. Carano RA and Filvaroff EH (2003) Angiogenesis and bone repair. Drug Discov Today 8, 980-989

23. Hsiao ST, Asgari A, Lokmic Z et al (2012) Comparative analysis of paracrine factor expression in human adult mesenchymal stem cells derived from bone marrow, adipose, and dermal tissue. Stem Cells Dev 21, 2189-2203

24. Konig J, Huppertz B, Desoye G et al (2012) Amnionderived mesenchymal stromal cells show angiogenic properties but resist differentiation into mature endothelial cells. Stem Cells Dev 21, 1309-1320

25. Fierro FA, O'Neal AJ, Beegle JR et al (2015) Hypoxic pre-conditioning increases the infiltration of endothelial cells into scaffolds for dermal regeneration pre-seeded with mesenchymal stem cells. Front Cell Dev Biol 3, 68

26. Oshima K, Nampei A, Matsuda M et al (2005) Adiponectin increases bone mass by suppressing osteoclast and activating osteoblast. Biochem Biophys Res Commun 331, 520-526

27. Williams GA, Wang Y, Callon KE et al (2009) In vitro and in vivo effects of adiponectin on bone. Endocrinology 150, 3603-3610

28. Yamaguchi N, Kukita T, Li YJ et al (2008) Adiponectin inhibits induction of TNF-alpha/RANKL-stimulated NFATc1 via the AMPK signaling. FEBS Lett 582, 451-456

29. Chen T, Wu YW, Lu H, Guo Y and Tang ZH (2015) Adiponectin enhances osteogenic differentiation in human adipose-derived stem cells by activating the APPL1-AMPK signaling pathway. Biochem Biophys Res Commun 461, 237-242

30. Li S, Huang L, Sun $Y$ et al (2015) Slit2 Promotes Angiogenic Activity Via the Robo1-VEGFR2-ERK1/2 Pathway in Both In Vivo and In Vitro Studies. Invest Ophthalmol Vis Sci 56, 5210-5217

31. Hammond CL and Schulte-Merker S (2009) Two populations of endochondral osteoblasts with differential sensitivity to Hedgehog signalling. Development 136, 3991-4000

32. Krum SA, Chang J, Miranda-Carboni G and Wang CY (2010) Novel functions for NFkappaB: inhibition of bone formation. Nat Rev Rheumatol 6, 607-611

33. Hu N, Feng C, Jiang Y, Miao Q and Liu H (2015) Regulative Effect of Mir-205 on Osteogenic Differentiation of Bone Mesenchymal Stem Cells (BMSCs): Possible Role of SATB2/Runx2 and ERK/MAPK Pathway. Int J Mol Sci 16, 10491-10506

34. Franceschi RT and Xiao G (2003) Regulation of the osteoblast-specific transcription factor, Runx2: responsiveness to multiple signal transduction pathways. J Cell Biochem 88, 446-454

35. Zhang D, Jiang M and Miao D (2011) Transplanted human amniotic membrane-derived mesenchymal stem cells ameliorate carbon tetrachloride-induced liver cirrhosis in mouse. PLoS One 6, e16789

36. Chen YJ, Chung MC, Jane Yao CC et al (2012) The effects of acellular amniotic membrane matrix on osteogenic differentiation and ERK1/2 signaling in human dental apical papilla cells. Biomaterials 33, 455-463

37. Kim KI, Park S and Im GI (2014) Osteogenic differentiation and angiogenesis with cocultured adipose-derived stromal cells and bone marrow stromal cells. Biomaterials 35, 4792-4804 